\title{
Robotic-Assisted Laparoscopic Side-to-Side Lateral Pancreaticojejunostomy
}

\author{
George Michel Eid • Fateh Entabi • \\ Andrew R. Watson • Brian S. Zuckerbraun • \\ Mark A. Wilson
}

Received: 2 July 2010 /Accepted: 21 March 2011 /Published online: 17 May 2011

(C) 2011 The Society for Surgery of the Alimentary Tract

Keywords Puestow · Pancreaticojejunostomy Robotic

\section{Introduction}

Side-to-side lateral pancreaticojejunostomy is commonly used to treat chronic pancreatitis. It is usually performed via a laparotomy due to the technical challenges of constructing a pancreatico-enteric anastomosis laparoscopically. The robotic system offers improved visualization and dexterity in fashioning such a complex anastomosis.

Electronic supplementary material The online version of this article (doi:10.1007/s11605-011-1495-9) contains supplementary material, which is available to authorized users.

G. M. Eid · F. Entabi · A. R. Watson • B. S. Zuckerbraun •

M. A. Wilson

Department of Surgery, University of Pittsburgh,

Pittsburgh, PA, USA

\section{G. M. Eid • M. A. Wilson}

Veterans Affairs Pittsburgh Healthcare System,

Pittsburgh, PA, USA

G. M. Eid $(\bowtie)$

Minimally Invasive Surgery Center,

Suite 390, 3380 Blvd of the Allies,

Pittsburgh, PA 15213, USA

e-mail: eidgm@upmc.edu

\section{Case Description}

A 58-year-old veteran was diagnosed with a gallstoneinduced chronic pancreatitis and malnutrition due to intractable abdominal pain. The patient had previously undergone laparoscopic cholecystectomy. Preoperative ERCP revealed an obstructing pancreatic duct stone in the head of the pancreas with associated upstream duct dilatation. The patient had failed multiple attempts to access and stent the pancreatic duct, including unsuccessful cannulation of the minor papilla. Pancreatic protocol CT scan demonstrated a well-developed pseudocyst in communication with the dilated pancreatic duct. The video explains in details the operative steps. He was discharged home on POD \#4. There were no complications during his hospitalization and at 6 months follow-up.

\section{Conclusion}

Robotic-assisted laparoscopic side-to-side lateral pancreaticojejunostomy is feasible and safe. The robot system offers the advantage of a true three-dimensional view based on a double optical system, in addition to a wide range of freedom of motion, which contributes to the feasibility of this advanced laparoscopic suturing. 